Document downloaded from:

http://hdl.handle.net/10251/83363

This paper must be cited as:

Andreu Estellés, C.; Castelló Palacios, S.; García Pardo, C.; Fornés Leal, A.; Vallés Lluch, A.; Cardona Marcet, N. (2016). Spatial In-Body Channel Characterization Using an Accurate UWB Phantom. IEEE Transactions on Microwave Theory and Techniques. 64(11):39954002. doi:10.1109/TMTT.2016.2609409.

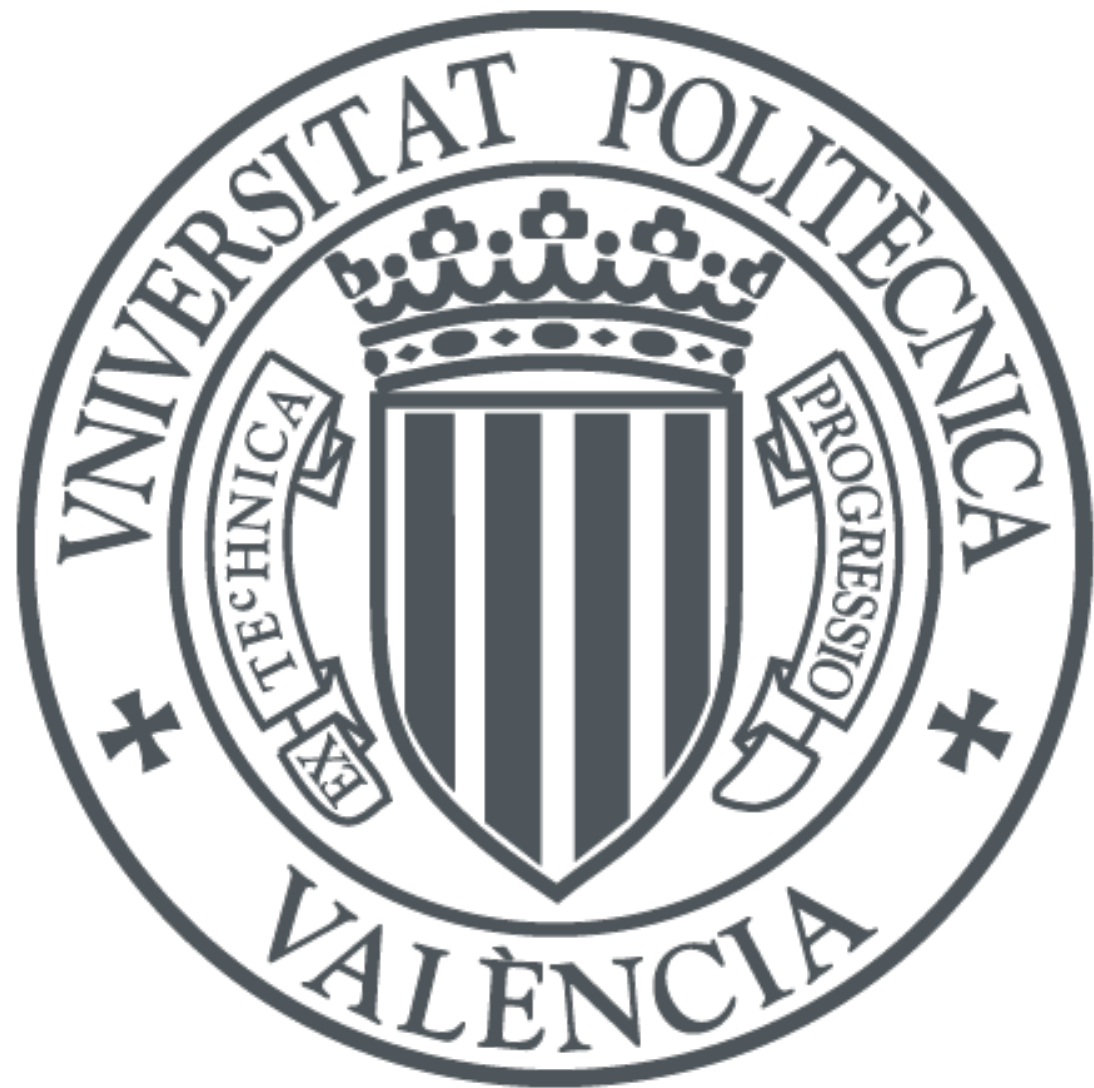

The final publication is available at

http://doi.org/10.1109/TMTT.2016.2609409

Copyright Institute of Electrical and Electronics Engineers (IEEE)

Additional Information

"(c) 2016 IEEE. Personal use of this material is permitted. Permission from IEEE must be obtained for all other users, including reprinting/ republishing this material for advertising or promotional purposes, creating new collective works for resale or redistribution to servers or lists, or reuse of any copyrighted components of this work in other works." 


\title{
Spatial In-Body Channel Characterization Using an Accurate UWB Phantom
}

\author{
Carlos Andreu, Sergio Castelló-Palacios, Concepcion Garcia-Pardo, Alejandro Fornes-Leal, \\ Ana Vallés-Lluch, and Narcís Cardona
}

\begin{abstract}
Ultra-wideband (UWB) systems have emerged as a possible solution for future wireless in-body communications. However, in-body channel characterization is complex. Animal experimentation is usually restricted. Furthermore, software simulations can be expensive and imply a high computational cost. Synthetic chemical solutions, known as phantoms, can be used to solve this issue. However, achieving a reliable UWB phantom can be challenging since UWB systems use a large bandwidth and the relative permittivity of human tissues are frequency dependent. In this paper, a measurement campaign within 3.1-8.5 GHz using a new UWB phantom is performed. Currently, this phantom achieves the best known approximation to the permittivity of human muscle in the whole UWB band. Measurements were performed in different spatial positions, in order to also investigate the diversity of the in-body channel in the spatial domain. Two experimental in-body to in-body (IB2IB) and in-body to on-body (IB2OB) scenarios are considered. From the measurements, new path loss models are obtained. Besides, the correlation in transmission and reception is computed for both scenarios. Our results show a highly uncorrelated channel in transmission for the IB2IB scenario at all locations. Nevertheless, for the IB2OB scenario, the correlation varies depending on the position of the receiver and transmitter.
\end{abstract}

Index Terms-Body area network, in-body communications, path loss, ultra-wideband (UWB) phantom, uncorrelated channel.

\section{INTRODUCTION}

W IRELESS body area networks allow the interconnection between independent nodes located either inside or over the body skin or further. With regard to in-body communications, establishing a proper link with a capsule endoscope [1] or with a pacemaker [2] are examples of technological advances achieved in the last decades. In spite of these healthcare developments, current standards for these kinds of communications do not allow high data rate wireless connections, which are common in the current telecommunication services [3]. The IEEE 802.15.6 standard

This work was supported by the Ministerio de Economía y Competitividad, Spain, under Grant TEC2014-60258-C2-1-R and Grant TEC2014-56469-REDT and by the European FEDER Funds.

C. Andreu, C. Garcia-Pardo, A. Fornes-Leal, and N. Cardona are with the iTEAM, Universitat Politècnica de València, 46022 Valencia, Spain (e-mail: caranes@iteam.upv.es; cgpardo@iteam.upv.es; alforlea@ iteam.upv.es; ncardona@iteam.upv.es).

S. Castelló-Palacios and A. Vallés-Lluch are with the Centre for Biomaterials and Tissue Engineering, Universitat Politècnica de València, 46022 Valencia, Spain (e-mail: sercaspa@etsii.upv.es; avalles@ter.upv.es).

Digital Object Identifier 10.1109/TMTT.2016.2609409 normalized the frequency bands for each propagation scenario. Concretely, the Medical Implant Communication Service (MICS) band was chosen to enable communication between in-body devices. The MICS frequency band (402-405 MHz) has a relatively good wave propagation conditions through biological tissues [4]. Nevertheless, this band is constrained to the use of narrowband systems. Particularly, the Industrial, Scientific and Medical (ISM) radio band (2400-2483.5 MHz) has been considered as a candidate in order to enhance the in-body communications in nextgeneration devices [5]. However, these multipurpose bands are widely used for WLAN and WPAN networks so that interferences between services could be produced. Thus, ultrawideband (UWB) frequency range has emerged as a future potential candidate for in-body communications [6]. The UWB frequency spectrum covers from 3.1 to $10.6 \mathrm{GHz}$ and its main benefits are high data rate, low power consumption, and the low-cost of its transceivers [7]. Nevertheless, the main drawback of UWB for in-body applications is the high attenuation of human body tissues, which dramatically increases with the increment in frequency [8]. Hence, an accurate UWB in-body channel characterization is relevant in order to establish a proper link between future UWB implantable nodes.

Implanting devices into human subjects in order to carry out propagation measurements is not possible due to ethical and physical reasons. Then, some research works have performed in vivo measurements using living animals such as pigs [9]-[11]. However, the high cost of each surgical procedure and the restrictions in animal experimentations due to ethical reasons are enough arguments to seek other solutions. In the literature, studies based on simulation measurements using human voxel models can be found [12]. Nevertheless, software simulations are not able to reproduce all the channel conditions. Besides, such software simulations can be an expensive solution as well as imply a high complex computational cost. On the other hand, chemical solutions, which emulate the electromagnetic behavior of human body tissues (i.e., complex relative permittivity), have been used in order to recreate the propagation through human tissues [5]. These mimicking materials are known as "phantoms" and have been used in previous propagation studies [13]. Nevertheless, the results obtained with these phantoms are far from the results obtained with living tissues [10]. Even though there are many phantoms that can reproduce the propagation medium with a relative accuracy within narrow frequency bands, only a few are provided for wide frequency bands. An aqueous solution of sugar has been reported as a UWB phantom [14], but its 
behavior is far from the real values reported in [8]. Other attempts have been made for wideband phantoms, but their accuracy decreases as frequency increases [15]. In particular, the reported phantoms of muscle for widebands show a quite bad approximation above $4-5 \mathrm{GHz}$ [16], mainly regarding the conductivity part.

The UWB frequency band aims at enhancing the current in-body applications. In order to improve the UWB channel performance, channel diversity of these systems can be exploited. For instance, current UWB systems have achieved accurate localization techniques [17]. These techniques benefit, among other features, from the diversity of the channel. These localization techniques can be influenced due to the correlation between receivers [18]. In the literature, many research works devoted to study the diversity performance of off-body systems can be found [19]. However, to the best of the authors' knowledge, not many studies about the UWB in-body channel diversity considering experimental scenarios are addressed in literature.

This paper aims at contributing to the UWB in-body channel characterization within $3.1-8.5 \mathrm{GHz}$ using a new realistic UWB phantom, which emulates the electromagnetic behavior of human muscle, as described in [8], with high accuracy in the whole frequency band considered [20]. Two in-body scenarios are considered depending on the location of the receiving antenna: in-body to in-body (IB2IB) and in-body to on-body (IB2OB) scenarios. Besides, a large amount of measurements has been provided by means of a novel spatial experimental setup using a 3-D automatic positioner. Hence, the path loss for both IB2IB and IB2OB can be characterized with high accuracy. Moreover, the correlation in transmission and reception is assessed from the measurements. For correlation in transmission, the channel impulse responses between different transmitting antenna positions are considered for a fixed position of the receiving antenna. The correlation at the receiving side is computed in an analogous form.

The remainder of this paper is organized as follows. In Section II, the measurement setups as well as the methodology are detailed. In Section III, the experimental path loss models for each scenario are presented. Section IV shows the correlation in transmission and reception for each scenario. Finally, Section V summarizes the conclusion obtained from this paper.

\section{Measurement Setup and Methodology}

\section{A. Experimental Scenarios}

With regard to the location of the antennas, two different scenarios were emulated.

1) IB2IB, where both antennas are considered to be located inside the human body.

2) IB2OB, where the in-body antenna is considered to be located inside the body, whereas the on-body antenna is placed over the human body surface.

\section{B. UWB Phantom}

Since the operation environment of these antennas is supposed to be the human body, in which it is not possible to

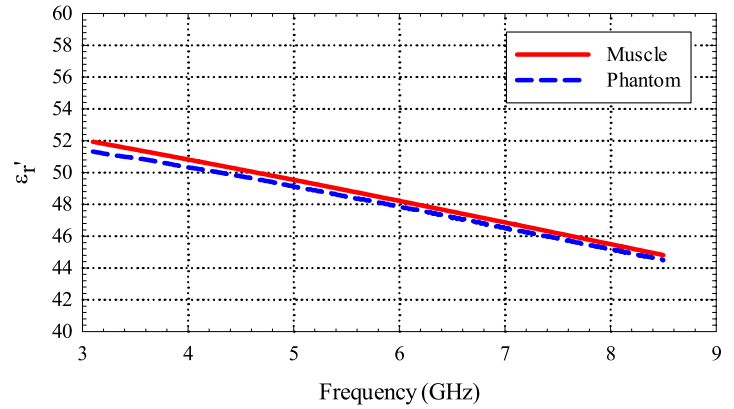

(a)

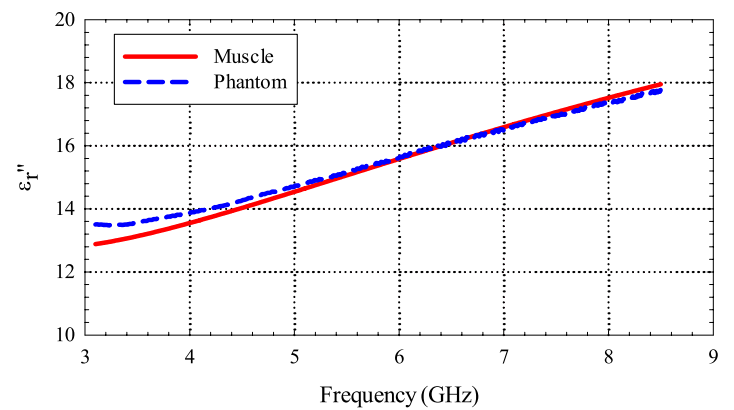

(b)

Fig. 1. Measured relative permittivity of the UWB phantom compared with the values of human muscle tissue reported in [8]. (a) Dielectric constant. (b) Loss factor.

test them, it becomes necessary to employ a tissue-equivalent medium. In this case, as muscle is the main human tissue or at least the future propagation medium for most wireless applications, a muscle-mimicking phantom was considered for our experiments. Keeping in mind that the antennas must be introduced and moved inside the phantom due to our scenarios, the required phantom should be liquid. For this purpose, the phantom proposed here is an aqueous solution of $54.98 \%$ acetonitrile and $1.07 \% \mathrm{NaCl}$, which owns the targeted conditions [20] and is easily prepared as follows: first, salt and acetonitrile were poured into a beaker in their corresponding amount, and then the required mass was filled with water and stirred for ten minutes until dissolution. This phantom was designed at $24{ }^{\circ} \mathrm{C}$. At such temperature, our solution reproduces the dielectric values of the human muscle that were characterized at body temperature, as reported in [8]. The variation of its dielectric properties with temperature is about \pm 0.08 units per degree celsius for the dielectric constant and \pm 0.11 units per degree for the loss factor. The key of this phantom is the use of acetonitrile, a polar molecule that gets dielectric constant values similar to those of muscle within a wide frequency band. Moreover, the addition of salt increases the loss factor so the tissue's behavior is better imitated in both parts of the complex relative permittivity

$$
\varepsilon_{r}=\varepsilon_{r}^{\prime}-j \varepsilon_{r}^{\prime \prime}
$$

where $\varepsilon_{r}^{\prime}$ is the dielectric constant and $\varepsilon_{r}^{\prime \prime}$ is the loss factor. In Fig. 1, the complex relative permittivity of our muscle phantom is compared with the reported measured values for a real muscle tissue [8].

This phantom is, to the best of the authors' knowledge, the most accurate imitation for the human muscle within the 


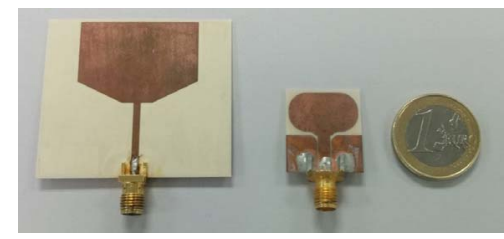

Fig. 2. UWB monopoles antennas used in both experimental scenarios.

UWB frequency band. In fact, it is worth mentioning that the maximum relative deviation from phantom values compared with a real tissue is $1.2 \%$ for the real part and $4.85 \%$ for the imaginary one, which occurs at $3 \mathrm{GHz}$.

\section{Antennas}

In order to perform a reliable UWB in-body measurement campaign, the antennas play a crucial role. They should have a compact structure in order to be implanted inside the human body in different locations as well as to be located over the body surface. Regarding an implanted transmitter, an antenna matching within the whole UWB frequency band is essential. Besides, an omnidirectional radiation pattern to communicate with a sensor array located around or inside the body should be achieved. In Fig. 2, the two UWB monopole antennas used in the measurement campaign are depicted. One antenna is approximately 2.2. times larger than the other. The small antenna, which is located at the center of Fig. 2, is an UWB monopole with microstrip feeding. The overall size is $23 \times 20 \mathrm{~mm}^{2}$. This miniaturized antenna was designed taking into account the human muscle permittivity values described in [8]. Thus, antenna matching as well as a quasiomnidirectional radiation pattern in the $x z$-plane within the UWB frequency range was achieved. Hereinafter, this antenna will be referred to as miniaturized antenna.

The large antenna also presents a quasi-omnidirectional radiation pattern in the $x z$-plane in the UWB frequency band. Further details of this antenna are given in [21]. Hereinafter, we refer to this antenna as a large antenna. This antenna can be used as an implanted transmitting antenna as well as an on-body or off-body receiver [10]. Besides, both in vivo and phantom-based measurement campaigns using the large antenna were performed [10], [22].

For the two scenarios previously described, the miniaturized antenna acted as the transmitting antenna, whereas the large antenna was the receiving one. Thus, the large antenna was fixed inside the phantom as the in-body antenna for the IB2IB case and outside the liquid phantom as the on-body antenna for the IB2OB scenario.

\section{Measurement Setup}

During measurements, the forward transmission coefficient $\left(S_{21}\right)$ was measured and processed for both scenarios. To perform this task, the antennas were connected to a vector network analyzer (VNA), which works up to $8.5 \mathrm{GHz}$. For both scenarios, the antennas were connected to ports 1 and 2 of the VNA by means of 2-m-length cables. In order to suppress the effect of the cables and connectors, the VNA was calibrated through a thru-calibration. The return loss of the antennas was measured previously in each measurement point in order to ensure antenna matching across the whole frequency band.

The output power of the VNA was set to $8 \mathrm{dBm}$ with a resolution bandwidth of $f_{\mathrm{IF}}=3 \mathrm{kHz}$. Measurements were performed from 3.1 to $8.5 \mathrm{GHz}$ with $N=1601$ resolution points resulting in a resolution frequency of $\Delta f=1.875 \mathrm{MHz}$. Five snapshots of the channel were taken in order to enhance the signal-to-noise ratio. The noise floor was different for each scenario, due to the change in antenna parameters of the receiving antenna. Thus, the noise levels in the IB2IB and IB2OB scenarios were at -95 and $-88 \mathrm{dBm}$, respectively.

The new UWB liquid phantom was poured into a polypropylene container. The container dimensions were $30 \times 30 \times$ $15 \mathrm{~cm}^{3}$ with a negligible wall thickness. The overall size of the box was chosen in order to mimic a cross section of the human torso. The dimensions in the $x y$ plane are widely used in simulation setups using voxel models [23]. Before being submerged into the UWB phantom, the antenna was covered with a layer of latex so that physical contact between antennas and liquid phantom was avoided. Latex rubber is widely used in health care environments. The antenna was carefully wrapped in order to remove the air as well as achieve an air-tight seal.

With the aim of performing channel measurements for different spatial positions, the miniaturized antenna was mounted on a robotic arm and moved to several $(x, y, z)$ locations inside the liquid phantom using a three-axis positioner. The error of this positioner is lower than $0.254 \mathrm{~mm} / \mathrm{cm}$ in each axis. In order to avoid disturbance during measurement time, the access to the room was restricted for everyone. Besides, possible interfering devices were switched OFF and the surrounding elements were at a minimum distance of $2 \mathrm{~m}$ far from the measurement setup. A fixed temperature of $24{ }^{\circ} \mathrm{C}$ during the measurements in both setups was assured by means of a thermostat located in the measurement room.

The miniaturized antenna was moved in steps of $\Delta x=$ $\Delta y=\Delta z=1 \mathrm{~cm}$ along the $x$-, $y$-, and $z$-axes, respectively. Considering the step resolution as well as the distance between each antenna and the internal container's walls, the distance between the antenna centers $d$ can be computed. During measurements, the positioner stopped in each location until the VNA had finished the acquisition. In order to control and synchronize the positioner and the VNA to perform the measurements, a software package was designed in Visual Studio.

Fig. 4 depicts the measurement setup particularized for the IB2IB scenario. The large antenna was placed in the middle of the $y$-axis, at $8 \mathrm{~cm}$ away from the container's wall located behind it ( $x$-axis), and at a height of $8.6 \mathrm{~cm}$ from the container's floor ( $z$-axis). The miniaturized antenna was located in a $x y z$ mesh of $11 \times 11 \times 2$ measurement points.

In the case of the IB2OB scenario, the large antenna was located over the external container's wall. In this scenario, two different setups were considered as follows.

1) Setup IB2OB.1: The on-body antenna (large antenna) was fixed in the center of the external container's wall, whereas the in-body antenna (miniaturized antenna) was located in a $x y z$ mesh of $19 \times 19 \times 3$ measurement points (see Fig. 3). 


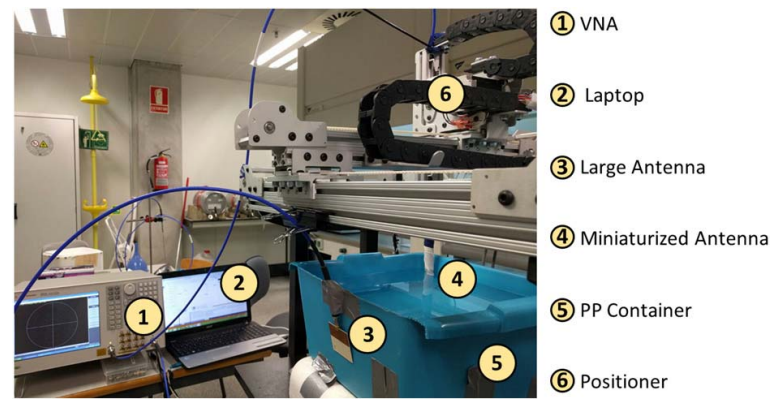

Fig. 3. Experimental measurement setup particularized for the IB2OB scenario.

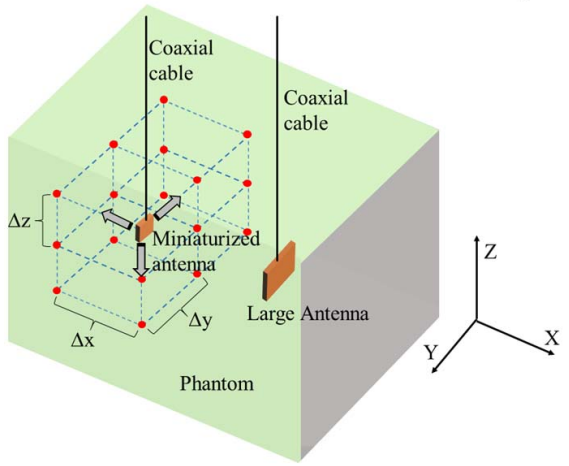

Fig. 4. Scheme of the $x y z$ mesh of measurement points traveled by the submerged antenna particularized for the IB2IB scenario.

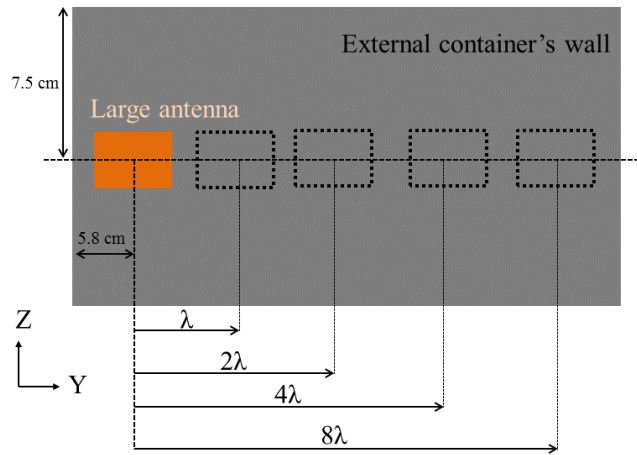

Fig. 5. Locations of on-body antenna in the experimental IB2OB.2 setup.

2) Setup IB2OB.2: The on-body antenna was located in an initial position (in orange in Fig. 5) and moved away $\lambda$, $2 \lambda, 4 \lambda$, and $8 \lambda$ in the $y$-axis, where $\lambda$ is the wavelength corresponding to the central frequency of the frequency band under analysis considering the propagation speed inside the phantom.

\section{E. Data Processing}

From the measurements, the path loss of each scenario was calculated. It should be highlighted that non-direct paths and wall reflections were highly attenuated due to the lossy propagation medium. Besides, only those $S_{21}$ samples $10 \mathrm{~dB}$ above the noise level were taken into account. As a consequence, the received power can be considered to be practically the direct path contribution. The path loss was computed as follows:

$$
\operatorname{PL}(\mathrm{dB})=-10 \log _{10}\left(\operatorname{mean}\left(|H(f)|^{2}\right)\right)
$$

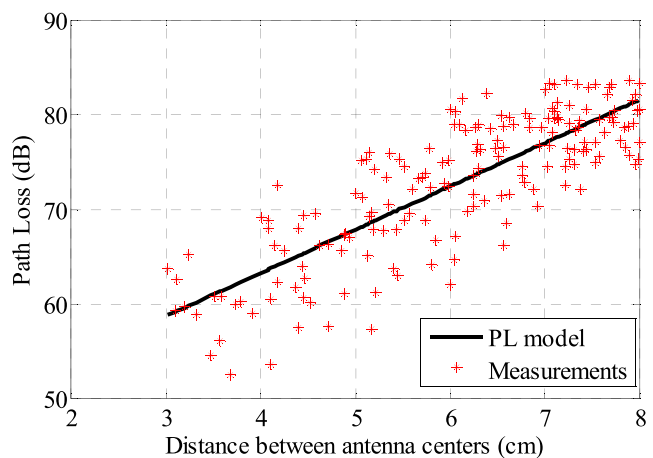

Fig. 6. Path loss as a function of distance between antenna centers $(d)$ in the experimental IB2IB scenario.

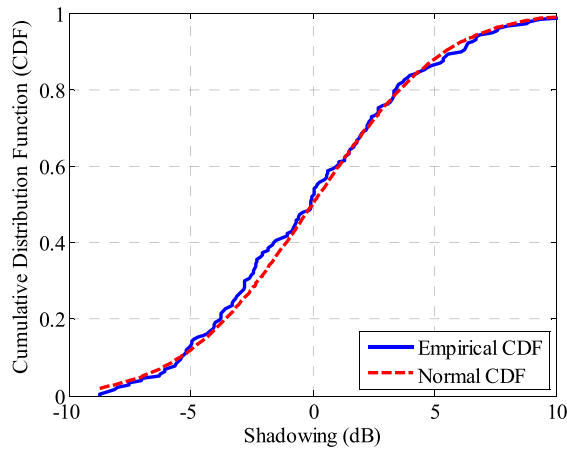

Fig. 7. Shadowing in the experimental IB2IB scenario.

with $H(f)$ being the frequency transfer function in $N$ resolution points computed as $H(f)=\left|S_{21}\right| e^{-j \varangle S_{21}}$, where $\left|S_{21}\right|$ and $\varangle S_{21}$ are module and phase in radians of the $S_{21}$, respectively.

The correlation coefficient between two different channel impulse responses, $h_{d_{1}}(\tau)$ and $h_{d_{2}}(\tau)$, was obtained as the maximum of the correlation as follows:

$$
\rho_{d_{1}, d_{2}}(\tau)=\frac{E\left[h_{d_{1}}(\tau) \cdot h_{d_{2}}^{*}(\tau)\right]}{\sqrt{E\left[\left|h_{d_{1}}(\tau)\right|^{2}\right] \cdot E\left[\left|h_{d_{2}}(\tau)\right|^{2}\right]}}
$$

where $E[]$ is the expectation operation value and $h_{d_{i}}(\tau)$ is the impulse channel response for a distance between antennas $d_{i}$, obtained from the IFFT of the $H(f)$ within $N$ points.

\section{PATH Loss Results}

In this section, the path loss models as a function of distance between antennas for both experimental setups are presented.

\section{A. IB2IB Scenario}

In Fig. 6, the path loss obtained from the measurements for the IB2IB scenario is shown. From Fig. 6, one can observe that the best fit is achieved with a linear approximation model as

$$
\mathrm{PL}(\mathrm{dB})=\mathrm{PL}_{0}+\alpha \cdot d(\mathrm{~cm})+X_{\sigma_{\mathrm{IB} 2 \mathrm{IB}}}
$$

where $\mathrm{PL}_{0}$ is the value of the path loss when the distance between antenna centers $d$ tends to $0, \alpha$ is a fitting parameter, and $X_{\sigma_{\mathrm{IB} 2 \mathrm{IB}}}$ is the shadowing term for IB2IB scenario. In this case, $\mathrm{PL}_{0}(\mathrm{~dB})=45.03 \mathrm{~dB}$ and $\alpha=4.57 \mathrm{~dB} / \mathrm{cm}$. Fig. 7 shows the cumulative distribution function of the measurements. It can be noted that the empirical model of shadowing can 


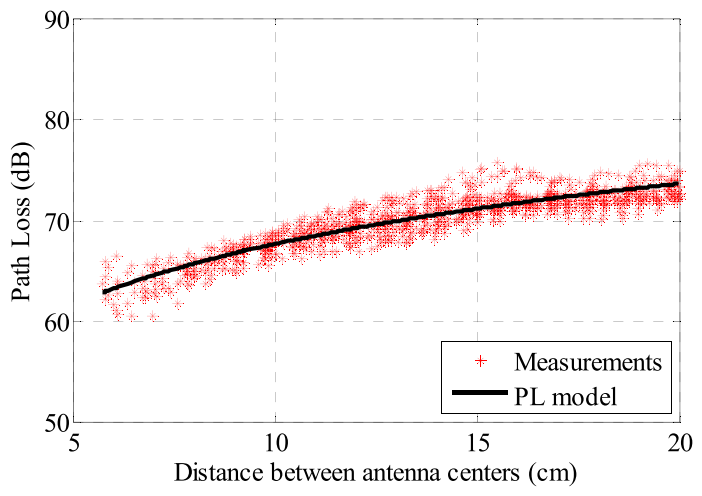

Fig. 8. Path loss as a function of distance between antenna centers $(d)$ in the experimental IB2OB scenario.

be fitted by means of a normal distribution with zero mean and standard deviation $\sigma=4.26(N(0, \sigma))$. It is important to note that the path loss is given from $d=3 \mathrm{~cm}$ to $d=8 \mathrm{~cm}$ because most of the measurements above $8 \mathrm{~cm}$ were at noise level.

As evidenced by our results, the path loss model has been well fitted by a linear approximation model. These results are in agreement with other research works, which were carried out in other frequency bands [5]. However, the set of measurements presented in this paper and those performed in [5] have been performed within different frequency bands and bandwidths. Indeed, there is a lack of measurements for IB2IB configurations within UWB frequency band in the literature.

\section{B. IB2OB Scenario}

The path loss values obtained for the IB2OB case are shown in Fig. 8. In this case, the path loss was fitted by means of a log-distance model since the measurement points follow this trending within the measured distances. The log-distance equation is given by the following expression:

$$
\mathrm{PL}(\mathrm{dB})=\mathrm{PL}_{0, d_{\mathrm{ref}}}+10 \gamma \log _{10}\left(\frac{d}{d_{\mathrm{ref}}}\right)+X_{\sigma_{\mathrm{IB} 2 \mathrm{OB}}}
$$

being $\mathrm{PL}_{0, d_{\text {ref }}}$ the path loss value in a reference distance $d_{\text {ref }}$ equal to $1 \mathrm{~cm}, \gamma$ the path loss exponent, $d$ the distance between antenna centers, and $X_{\sigma_{\mathrm{IB} 2 \mathrm{OB}}}$ the shadowing term for the IB2OB scenario. In this case, the parameters of the log-distance equation were $\mathrm{PL}_{0, d_{\mathrm{ref}}}=47.84 \mathrm{~dB}$ and $\gamma=$ 1.98. From Fig. 9, one can also observe that the shadowing also follows a normal distribution with zero mean and $\sigma=1.2$.

As mentioned above, the path loss model obtained from our measurements follows a logarithmic trend as the distance between antennas increases. Chávez-Santiago et al. [6] proposed a path loss model from 0 to $12 \mathrm{~cm}$ within 1 to $6 \mathrm{GHz}$ in a heterogeneous medium by means of a software simulation. As it can be checked, our path loss model is similar to that obtained in [6] within the same distance range. However, our IB2OB path loss model is given for a higher range of distances. Moreover, their path loss model is defined within a lower frequency band (1-6 GHz). Besides, our path loss model can also be compared with that obtained from in vivo measurements [22]. Although our path loss model is defined

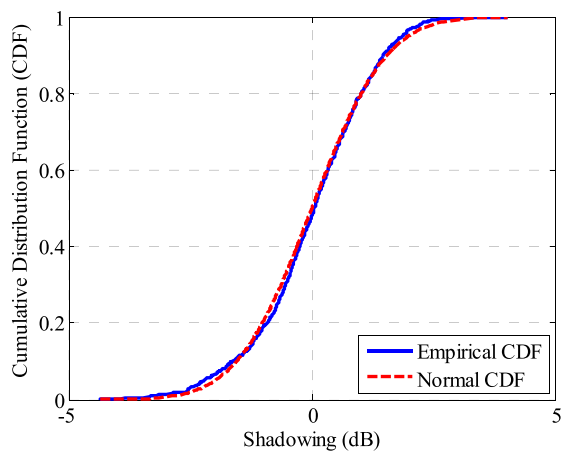

Fig. 9. Shadowing in the experimental IB2OB scenario.

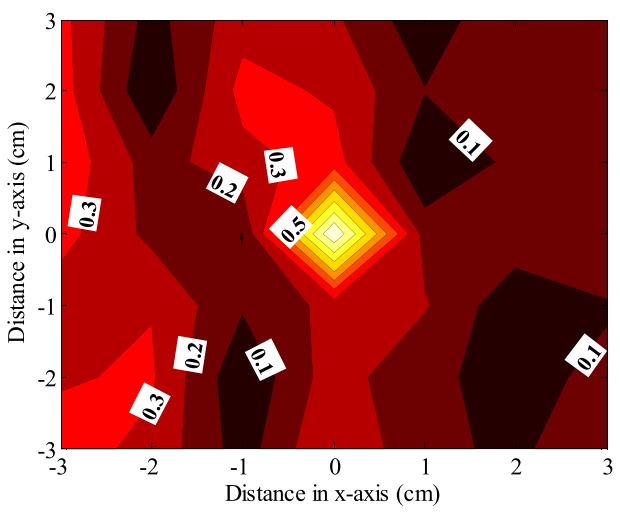

Fig. 10. Correlation coefficients at $z=0$ in the experimental IB2IB scenario.

in distance above $12 \mathrm{~cm}$, the trend of our path loss model and the aforementioned path loss is comparable considering the same range of values for the distance between antennas. However, absolute values of the path loss are different due to the different antennas used in both experiments.

Garcia-Pardo et al. [10] compared phantom-based and in vivo measurements in an IB2OB scenario using the phantom described in [14]. As can be seen, the propagation losses in [10] are higher than those obtained using the new phantom. That is by the fact that the dielectric values of the new phantom are close to those obtained by Gabriel [8]. On the contrary, the phantom proposed in [14] overestimates the losses as concluded in [10].

\section{Correlation Coefficients}

The diversity of the channel in transmission and reception is assessed throughout this section. For this purpose, the correlation coefficients in each scenario are computed.

\section{A. $I B 2 I B$}

For IB2IB scenario, the correlation in transmission for transmitters located in the same $x y$ plane is shown in Fig. 10. In this case, the position of the reference impulse channel response was located at the center of the $x y$ plane at $z=0$. The distance between antenna centers at this reference locationdenoted by subscript $d_{1}$ in (2)-was $d_{1}=5.77 \mathrm{~cm}$. From Fig. 10, it can be deduced that the correlation decreases rapidly as the distance increases in both $x$ - and $y$-axes. Moreover, one can observe that the correlation drops below 0.3 , when the transmitting antenna location is more than $1 \mathrm{~cm}$ away 


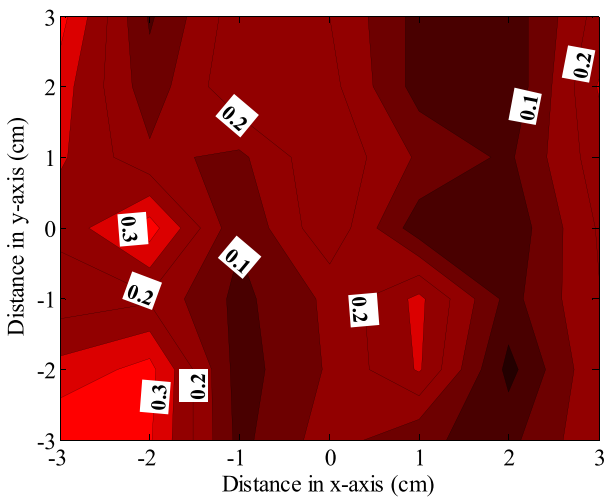

Fig. 11. Correlation coefficients at a distance of $\Delta z$ from $z=0$ in the experimental IB2IB scenario.

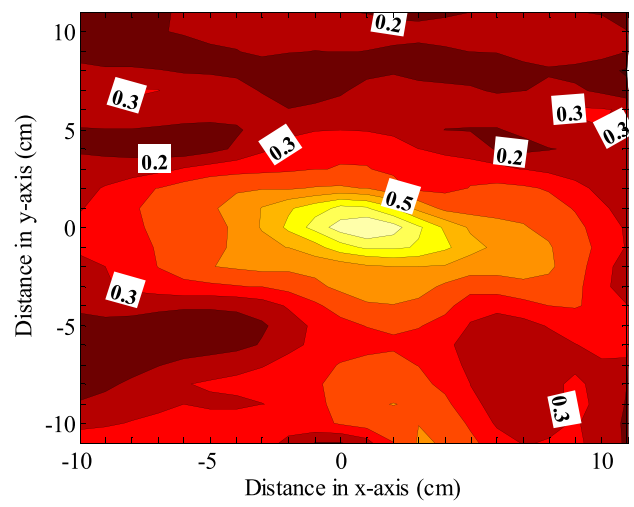

Fig. 12. Correlation coefficients at $z=0$ in the experimental IB2OB.1 scenario.

from the location of the reference transmitter. It should be mentioned that the resolution distance between the measured transmitter positions is $1 \mathrm{~cm}$.

Fig. 11 shows the correlation in transmission for transmitters located at different $x y$ planes. The reference channel impulse response was correlated with those located in an upper plane. This upper plane was separated at a distance $\Delta z(1 \mathrm{~cm})$. It can be noted that the correlation is lower than 0.3 for all the positions in the upper plane. Hence, it can be concluded that the channel is highly uncorrelated at different antenna heights.

\section{B. $I B 2 O B$}

In the IB2OB scenario, the correlation in transmission was calculated in the same manner as in the previous case, considering the setup IB2OB.1. Thus, the reference position was located again at the center of the $x y$ plane at $z=0$, being the distance between antenna centers in this case of $d_{1}=14.83 \mathrm{~cm}$. Thus, the values of the correlation coefficients are shown in Fig. 12. As can be observed, the correlation in the same $x y$ plane is high in those positions close to the reference one. Thus, the IB2OB channel can be considered to be uncorrelated for transmitting positions separated approximately more than $5 \mathrm{~cm}$ in each axis.

Fig. 13 shows the correlation in transmission when the position between transmitters was $\Delta z(1 \mathrm{~cm})$ and $2 \Delta z(2 \mathrm{~cm})$ from the reference position in the $z$-axis. It can be noted

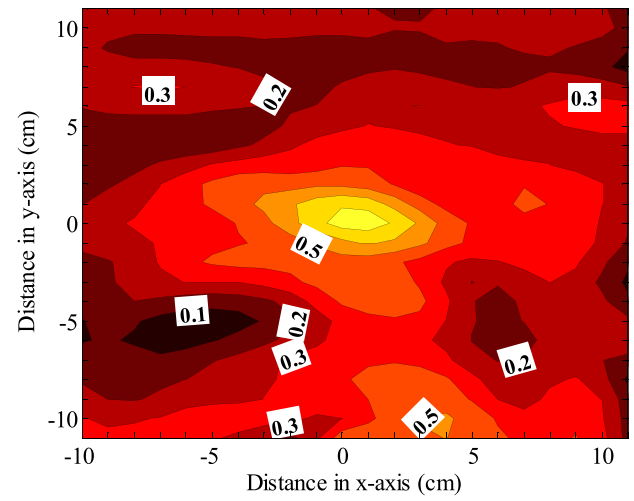

(a)

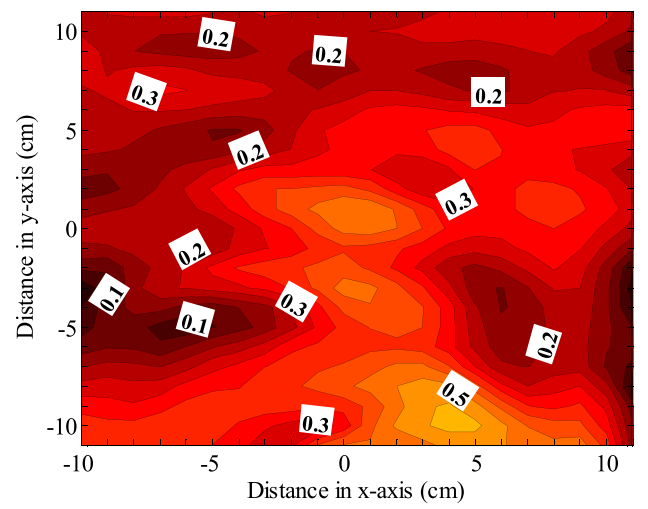

(b)

Fig. 13. Correlation coefficientes at a distance of (a) $z=\Delta z$ and (b) $z=2 \Delta z$ from the plane $z=0$ in experimental IB2OB.1 scenario.

TABLE I

Correlation COEFFICIENTS ALONG THE $x$-AXIS IN THE IB2OB. 2 SCENARIO

\begin{tabular}{ccccc}
\hline \hline & $\lambda$ & $2 \lambda$ & $4 \lambda$ & $8 \lambda$ \\
\hline$-5 \Delta \mathrm{x}$ & 0.62 & 0.37 & 0.35 & 0.29 \\
$-4 \Delta \mathrm{x}$ & 0.55 & 0.36 & 0.28 & 0.26 \\
$-3 \Delta \mathrm{x}$ & 0.54 & 0.35 & 0.25 & 0.25 \\
$-2 \Delta \mathrm{x}$ & 0.57 & 0.36 & 0.26 & 0.28 \\
$-\Delta \mathrm{x}$ & 0.63 & 0.42 & 0.29 & 0.24 \\
0 & 0.65 & 0.46 & 0.27 & 0.18 \\
$\Delta \mathrm{x}$ & 0.66 & 0.46 & 0.22 & 0.17 \\
$2 \Delta \mathrm{x}$ & 0.67 & 0.44 & 0.19 & 0.18 \\
$3 \Delta \mathrm{x}$ & 0.69 & 0.45 & 0.20 & 0.16 \\
$4 \Delta \mathrm{x}$ & 0.71 & 0.47 & 0.23 & 0.14 \\
$5 \Delta \mathrm{x}$ & 0.73 & 0.49 & 0.22 & 0.14 \\
\hline
\end{tabular}

that the correlation is lower as the distance between planes in the $z$-axis increases. This effect can be observed from Fig. 13(a) and (b). On the one hand, in Fig. 13(a), the correlation was reduced with respect to the case of $z=0$ for all positions. On the other hand, in Fig. 13(b), where the separation distance in the $z$-axis was $2 \Delta z$, uncorrelated signals were found whatever the separation of transmitting positions.

In order to evaluate the correlation in reception, the setup IB2OB.2 was considered (see Section II-D). Table I shows the correlation coefficients for different receiving antenna positions (see Fig. 5) as a function of different transmitting antenna locations along the $x$-axis. It should be mentioned that for location 0 , the transmitting antenna was located again 
TABLE II

Correlation CoefFicients Along the $y$-AXIS IN THE IB2OB. 2 SCENARIO

\begin{tabular}{ccccc}
\hline \hline & $\lambda$ & $2 \lambda$ & $4 \lambda$ & $8 \lambda$ \\
\hline$-5 \Delta \mathrm{y}$ & 0.67 & 0.46 & 0.22 & 0.18 \\
$-4 \Delta \mathrm{y}$ & 0.68 & 0.48 & 0.24 & 0.17 \\
$-3 \Delta \mathrm{y}$ & 0.66 & 0.48 & 0.21 & 0.17 \\
$-2 \Delta \mathrm{y}$ & 0.58 & 0.42 & 0.21 & 0.19 \\
$-\Delta \mathrm{y}$ & 0.54 & 0.35 & 0.20 & 0.18 \\
0 & 0.53 & 0.37 & 0.24 & 0.11 \\
$\Delta \mathrm{y}$ & 0.54 & 0.41 & 0.32 & 0.10 \\
$2 \Delta \mathrm{y}$ & 0.53 & 0.39 & 0.34 & 0.08 \\
$3 \Delta \mathrm{y}$ & 0.50 & 0.42 & 0.31 & 0.17 \\
$4 \Delta \mathrm{y}$ & 0.47 & 0.41 & 0.32 & 0.10 \\
$5 \Delta \mathrm{y}$ & 0.44 & 0.37 & 0.29 & 0.09 \\
\hline
\end{tabular}

at the center position of the $x y$ plane. The rest of locations corresponds to $\Delta x$ steps from location 0 of the transmitter. From Table I, one can deduce that the correlation coefficients are lower as the distance between receiving antennas is higher. Besides, the correlation is below 0.5 for a separation distance between receivers above $\lambda$. It can be noted that the correlation decreases rapidly as the distance between receivers increases as concluded in [24].

Likewise, Table II shows the correlation coefficients between receiving antennas when the position of the transmitting antenna was varied in the $y$-axis. In this case, the correlation also decreases when the distance between receiving antennas increases. As in the previous case, the correlation is below 0.5 for a distance above $\lambda$, whatever be the position of the transmitting antenna in the $y$-axis.

\section{Conclusion}

In this paper, a set of phantom-based measurements within 3.1-8.5 GHz is presented. As a novelty, a new UWB phantom that mimics the permittivity values of human muscle tissue in the whole UWB frequency band with high accuracy was used. Using this new phantom, the IB2IB and IB2OB scenarios were considered. In order to perform the measurements, an automatic three-axis positioner was used. Thus, a large amount of measurements with high location accuracy were obtained in order to characterize the UWB in-body channel. Two path loss models for the IB2IB and IB2OB scenarios were obtained. In the IB2IB case, the path loss model, which best fitted the measurements for distances between antennas ranging from 3 to $8 \mathrm{~cm}$, was a linear model. In contrast, the path loss was fitted by a log-distance model from 5.5 to $20 \mathrm{~cm}$ for the IB2OB scenario.

The diversity of the UWB in-body channel was also investigated by means of the calculation of the correlation coefficients for both scenarios. In the IB2IB case, the correlation in transmission was below 0.5 regardless of the distance to the reference transmitter. For the IB2OB scenario, our findings revealed that the use of two receiving antennas separated more than $\lambda$ is essential to obtain uncorrelated channel impulse responses in reception. With regard to the correlation coefficients between transmitting antennas, it is lower when the transmitters are located in different $z$ planes for the same $x y$ position. For future work, it would be crucial to manufacture other human phantoms such as fat and skin. In this manner, the effects of the heterogeneity of the human tissues on UWB channel characterization will be checked. These results could be contrasted with simulations using human voxel models.

\section{REFERENCES}

[1] G. Iddan, G. Meron, A. Glukhovsky, and P. Swain, "Wireless capsule endoscopy," Nature, vol. 405, p. 417, May 2000.

[2] H. Burri and D. Senouf, "Remote monitoring and follow-up of pacemakers and implantable cardioverter defibrillators," Europace, vol. 11, no. 6, pp. 701-709, Jun. 2009.

[3] IEEE Standard for Local and Metropolitan Area Networks-Part 15.6: Wireless Body Area Networks, IEEE Standard 802.15.6-2012, 2012, pp. 1-271.

[4] W. G. Scanlon, B. Burns, and N. E. Evans, "Radiowave propagation from a tissue-implanted source at $418 \mathrm{MHz}$ and $916.5 \mathrm{MHz}$," IEEE Trans. Biomed. Eng., vol. 47, no. 4, pp. 527-534, Apr. 2000

[5] R. Chávez-Santiago et al., "Experimental path loss models for inbody communications within 2.36-2.5 GHz," IEEE J. Biomed. Health Informat., vol. 19, no. 3, pp. 930-937, May 2015.

[6] R. Chávez-Santiago et al., "Propagation models for IEEE 802.15.6 standardization of implant communication in body area networks," IEEE Commun. Mag., vol. 51, no. 8, pp. 80-87, Aug. 2013.

[7] X. Shen, M. Guizani, R. C. Qiu, and T. Le-Ngoc, Eds., Ultra-Wideband Wireless Communications and Networks. Hoboken, NJ, USA: Wiley, 2007.

[8] C. Gabriel, "Compilation of the dielectric properties of body tissues at RF and microwave frequencies," Brooks Air Force, San Antonio, TX, USA, Tech. Rep. N.AL/OE-TR-1996-0037, 1996.

[9] P. A. Floor et al., "In-body to on-body ultrawideband propagation model derived from measurements in living animals," IEEE J. Biomed. Health Informat., vol. 19, no. 3, pp. 938-948, May 2015.

[10] C. Garcia-Pardo, R. Chávez-Santiago, N. Cardona, and I. Balasingham, "Experimental UWB frequency analysis for implant communications," in Proc. 37th Annu. Int. Conf. IEEE Eng. Med. Biol. Soc. (EMBC), Aug. 2015, pp. 5457-5460.

[11] H. Xie et al., "Shear wave dispersion ultrasound vibrometry (SDUV) on an ultrasound system: In vivo measurement of liver viscoelasticity in healthy animals," in Proc. IEEE Ultrason. Symp., Oct. 2010, pp. 912-915.

[12] A. Khaleghi, R. Chávez-Santiago, and I. Balasingham, "Ultra-wideband statistical propagation channel model for implant sensors in the human chest," IET Microw. Antennas Propag., vol. 5, no. 15, pp. 1805-1812, Dec. 2011.

[13] R. Chávez-Santiago, C. García-Pardo, A. Fornes-Leal, A. Vallés-Lluch, I. Balasingham, and N. Cardona, "Ultra wideband propagation for future in-body sensor networks," in Proc. IEEE 25th Annu. Int. Symp. Pers., Indoor, Mobile Radio Commun. (PIMRC), Sep. 2014, pp. 2160-2163.

[14] H. Yamamoto, J. Zhou, and T. Kobayashi, "Ultra wideband electromagnetic phantoms for antennas and propagation studies," IEICE Trans. Fundam. Electron., Commun. Comput. Sci., vol. E91-A, no. 11, pp. 3173-3182, Nov. 2008.

[15] M. Lazebnik, E. L. Madsen, G. R. Frank, and S. C. Hagness, "Tissue-mimicking phantom materials for narrowband and ultrawideband microwave applications," Phys. Med. Biol., vol. 50, no. 18, pp. 4245-4258, Sep. 2005.

[16] T. Yilmaz, R. Foster, and Y. Hao, "Broadband tissue mimicking phantoms and a patch resonator for evaluating noninvasive monitoring of blood glucose levels," IEEE Trans. Antennas Propag., vol. 62, no. 6, pp. 3064-3075, Jun. 2014

[17] S. Gezici et al., "Localization via ultra-wideband radios: A look at positioning aspects for future sensor networks," IEEE Signal Process. Mag., vol. 22, no. 4, pp. 70-84, Jul. 2005.

[18] B. Moussakhani, J. T. Flam, S. Stoa, I. Balasingham, and T. Ramstad, "On localisation accuracy inside the human abdomen region," IET Wireless Sensor Syst., vol. 2, no. 1, pp. 9-15, Mar. 2012

[19] M. Marinova et al., "Diversity performance of off-body MB-OFDM UWB-MIMO," IEEE Trans. Antennas Propag., vol. 63, no. 7, pp. 3187-3197, Jul. 2015.

[20] N. Cardona, S. Castelló-Palacios, A. Fornes-Leal, C. Garcia-Pardo, and A. Vallés-Lluch, "Modelo sintético de tejidos biológicos para la evaluación de la transmisión inalámbrica de ondas electromagnéticas," (in Spanish), ES Patent 2575731, Dec. 22, 2015. 
[21] C. Tarin, P. Marti, L. Traver, N. Cardona, J. A. Diaz, and E. Antonino, "UWB channel measurements for hand-portable devices: A comparative study," in Proc. IEEE 18th Int. Symp. Pers., Indoor Mobile Radio Commun., Sep. 2007, pp. 1-5.

[22] C. Garcia-Pardo et al., "Experimental ultra wideband path loss models for implant communications," in Proc. 27th IEEE Int. Symp. Pers., Indoor Mobile Radio Commun., Valencia, Spain, Sep. 2016, pp. $1350-1355$.

[23] M. Kawasaki and R. K. Kohno, "A toa based positioning technique of medical implanted devices," in Proc. 3rd Int. Symp. Med. Inf. Commun. Technol. (ISMICT), Montreal, QC, Canada, 2009, pp. 1-5.

[24] J. Shi, D. Anzai, and J. Wang, "Channel modeling and performance analysis of diversity reception for implant UWB wireless link," IEICE Trans. Commun., vol. E95-B, no. 10, pp. 3197-3205, 2012. 